\title{
Targeting the myostatin signaling pathway to treat muscle loss and metabolic dysfunction
}

\author{
Se-Jin Lee ${ }^{1,2}$ \\ 'The Jackson Laboratory for Genomic Medicine, Farmington, Connecticut, USA. ${ }^{2}$ University of Connecticut School of Medicine, Department of Genetics and Genome Sciences, Farmington, Connecticut, USA.
}

\begin{abstract}
Since the discovery of myostatin (MSTN; also known as GDF-8) as a critical regulator of skeletal muscle mass in 1997, there has been an extensive effort directed at understanding the cellular and physiological mechanisms underlying MSTN activity, with the long-term goal of developing strategies and agents capable of blocking MSTN signaling to treat patients with muscle loss. Considerable progress has been made in elucidating key components of this regulatory system, and in parallel with this effort has been the development of numerous biologics that have been tested in clinical trials for a wide range of indications, including muscular dystrophy, sporadic inclusion body myositis, spinal muscular atrophy, cachexia, muscle loss due to aging or following falls, obesity, and type 2 diabetes. Here, I review what is known about the MSTN regulatory system and the current state of efforts to target this pathway for clinical applications.
\end{abstract}

\section{Introduction}

Myostatin (MSTN; also known as GDF-8) is a secreted signaling molecule that was originally identified in a screen for new members of the TGF- $\beta$ superfamily (1). MSTN's function was revealed by gene targeting studies, which showed that mice carrying a deletion of the Mstn gene exhibit dramatic increases in skeletal muscle mass throughout the body, with individual muscles growing to about twice the normal size. Subsequent studies showed that the MSTN gene has been strongly conserved through evolution, with the amino acid sequence of mature MSTN being identical in species as divergent as humans and turkeys (2). The function of MSTN as a negative regulator of skeletal muscle mass has also been strongly conserved, as targeted or naturally occurring mutations in cattle (2-4), sheep (5), dogs (6), rabbits (7), rats (8), swine (9), goats (10), and humans (11) have all been shown to lead to increased muscling. MSTN's discovery launched an extensive effort directed at understanding the molecular and cellular mechanisms underlying MSTN activity with the long-term goal of developing strategies to target this signaling pathway for therapeutic applications. A PubMed search for the term "myostatin" now identifies over 3000 papers addressing the regulation and function of MSTN in both normal and pathological states, and extensive preclinical studies looking at the effects of MSTN inhibition in mouse models of various human diseases have supported the development of MSTN inhibitors for clinical use. To date, at least nine biotechnology and pharmaceutical companies have developed MSTN inhibitors that have been tested in clinical trials for a wide range of indications characterized by muscle loss, including 22 trials that have reached

Conflict of interest: SJL is the inventor on the following provisional patent application: "Therapeutics targeting transforming growth factor beta family signaling" (serial no. 63/113,575)

Copyright: (c) 2021, American Society for Clinical Investigation.

Reference information: J Clin Invest. 2021;131(9):e148372.

https://doi.org/10.1172/JCl148372. phase II or phase III. Here I provide an overview of the drug development activities focused on MSTN and discuss some of the issues and challenges in developing the most effective strategies to target this pathway for clinical applications.

\section{The MSTN regulatory system}

Key to the development of many MSTN-targeting therapeutic strategies and agents has been the elucidation of many of the regulatory and signaling components underlying MSTN activity. As shown in Figure 1, MSTN is synthesized in a precursor form, which is cleaved by furin proteases to generate an $\mathrm{N}$-terminal propeptide and a disulfide-linked C-terminal dimer, which is the actual signaling molecule (12). Following proteolytic processing, the propeptide remains noncovalently bound to MSTN (12), and a variety of both in vitro and in vivo studies have shown that the propeptide maintains MSTN in an inactive, latent state. Specifically, purified propeptide is capable of blocking MSTN activity in vitro in both reporter gene and receptor binding assays $(12,13)$, and transgenic overexpression of the propeptide in skeletal muscle was shown to phenocopy the Mstn loss-of-function mutation in terms of increased muscle mass (12). Moreover, MSTN has been shown to circulate in the blood in an inactive form (14), and affinity purification of MSTN complexes from the blood using a monoclonal antibody directed against MSTN identified the propeptide as a major MSTN binding protein in vivo (15); in fact, based on quantification of the relative molar amounts of MSTN and propeptide in these preparations, it was estimated that the majority of circulating MSTN is present in a complex with its propeptide.

A key question has been how MSTN is activated from this latent complex. Early studies showed that latent MSTN can be activated artificially by treatment with either acid (14) or heat (16). Subsequent studies showed that latent MSTN can also be activated by proteolytic cleavage of the propeptide by members of the BMP-1/ tolloid family of metalloproteases (16). All four members of this protease family, BMP-1, TLD, TLL-1, and TLL-2, are capable of cleav- 


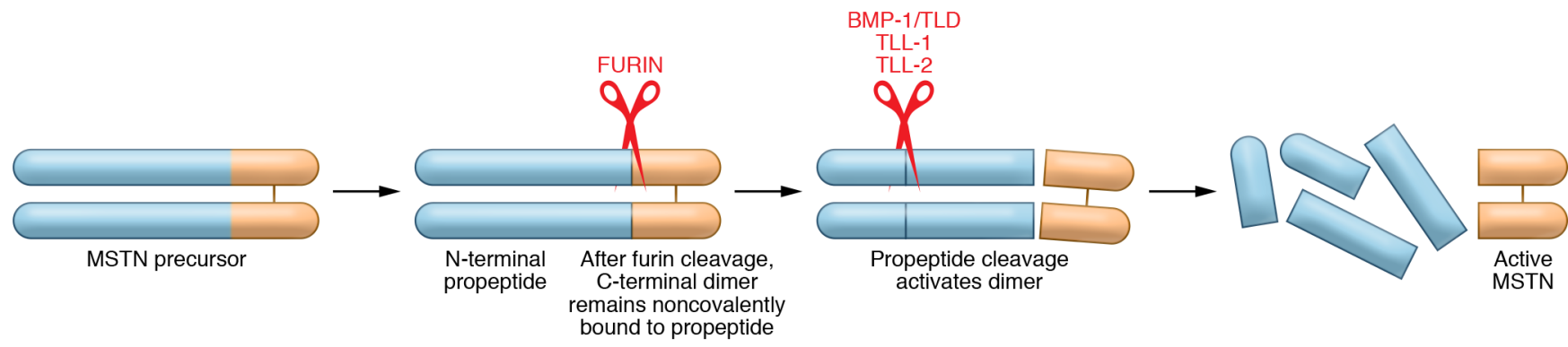

Figure 1. Processing of MSTN. The MSTN precursor protein is cleaved by furin proteases to generate an N-terminal propeptide (blue) and a C-terminal dimer (orange), which is the actual signaling molecule. The propeptide remains noncovalently bound to the C-terminal dimer and maintains MSTN in an inactive, latent state. Latent MSTN is activated by proteolytic cleavage of the propeptide immediately $\mathrm{N}$-terminal to aspartate residue 76 by members of the BMP-TLD family of metalloproteases, which frees up the C-terminal dimer to bind to cell surface receptors. Adapted with permission from the Annual Review of Cell and Developmental Biology (112).

ing the propeptide at a site immediately $\mathrm{N}$-terminal to aspartate 76 , thereby causing dissociation and activation of the latent complex (Figure 1). Two lines of genetic evidence have suggested that this is the major mechanism by which MSTN is activated in vivo. First, mice engineered to carry a point mutation changing aspartate 76 to alanine (D76A), which renders the propeptide resistant to BMP1 cleavage in vitro, exhibit increases in muscle mass approaching those seen in Mstn-null mice (17). A striking finding was that circulating levels of MSTN are increased dramatically in mice homozygous for D76A, and yet D76A mice exhibit a phenotype reminiscent of that seen in mice completely lacking MSTN, reflecting the fact that the aspartate-to-alanine change renders MSTN unable to be activated from its latent state in vivo. Second, mice carrying a lossof-function mutation in Tll2 also exhibit increases in muscle mass, although the relatively small magnitude of these increases suggests that TLL-2's function is at least partially redundant with that of one or more other BMP-1/tolloid proteases (17).

MSTN is regulated extracellularly by multiple other binding proteins in addition to the propeptide (Figure 2). One of these is follistatin (FST), which is capable of binding multiple TGF- $\beta$ family members (18-22). FST is also capable of binding MSTN and preventing it from binding to its receptors (12), and results of genetic studies in mice support a key role for FST in regulating MSTN activity in vivo. In particular, mice overexpressing FST in skeletal muscle exhibit dramatic muscle growth, consistent with inhibition of the MSTN pathway (12), and newborn $\mathrm{Fst}^{-/-}$mice (23) as well as adult $\mathrm{Fst}^{+/-}$mice (24) have reduced muscle mass, consistent with overactivity of MSTN ( $F s t^{-/-}$mice are not viable). Affinity purification of MSTN from the blood of both mice and humans has also identified an FST-related protein, FSTL-3 (also called FLRG), as another binding partner for MSTN (15). FSTL-3 shares structural similarity to FST in that it contains follistatin domains that mediate ligand binding (25), and like FST, FSTL-3 is capable of inhibiting MSTN activity in vitro in reporter gene assays (15). Overexpression studies have documented that FSTL-3 can also increase muscle mass in vivo $(26,27)$, but Fstl3-null mice appear to have normal muscle mass $(24,28)$.

A third binding protein containing a follistatin domain, namely growth and differentiation factor-associated serum protein-1 (GASP-1), was also identified from blood in these affinity purification studies (29). GASP-1 (also called WFIKKN2), origi- nally identified as a protein containing multiple domains associated with protease inhibitors (30), can bind MSTN and inhibit its activity in vitro in reporter gene (29) as well as receptor binding assays (31). A related protein, GASP-2 (also called WFIKKN1), is also capable of binding and inhibiting $\operatorname{MSTN}(31,32)$, although GASP-2 was not detected as one of the proteins bound to MSTN in the blood $(15,29)$. GASP-1 also appears to be capable of blocking MSTN in vivo, as overexpression of GASP-1 in skeletal muscle has been shown to induce increases in muscle mass and grip strength (27). Moreover, mice carrying targeted deletions of Gasp1 and/or Gasp2 exhibit reduced muscle mass, impaired muscle regeneration ability, and fiber type shifts, all of which are consistent with their roles in inhibiting MSTN in vivo (31).

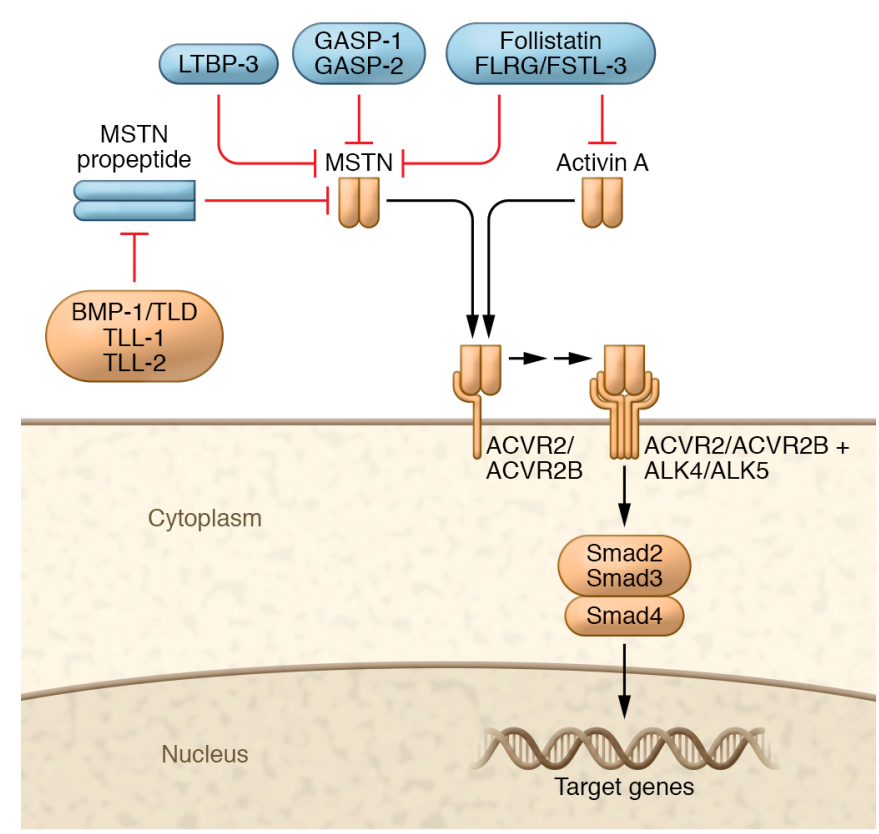

Limits muscle growth

Enhances muscle growth

Figure 2. Components of the MSTN regulatory system. Components shown in orange act positively in the pathway to limit muscle growth. Components shown in blue act negatively in the pathway to induce muscle growth. Adapted with permission from Immunology, Endocrine and Metabolic Agents in Medicinal Chemistry (114). 
Table 1. MSTN inhibitors tested in clinical trials

$\begin{array}{lccc}\text { Company } & \text { Drug } & \text { Type } & \text { Ref. } \\ \text { Wyeth/Pfizer } & \text { MYO-029 } & \text { Anti-MSTN monoclonal antibody } & 115 \\ \text { Pfizer } & \text { Domagrozumab } & \text { Anti-MSTN monoclonal antibody } & 42,43 \\ \text { Eli Lilly } & \text { LY2495655 } & \text { Anti-MSTN monoclonal antibody } & 44 \\ \text { Regeneron } & \text { REGN1033 } & \text { Anti-MSTN monoclonal antibody } & 45 \\ \text { Amgen } & \text { AMG-745 } & \text { Anti-MSTN peptibody } & 46 \\ \text { Atara } & \text { PINTA-745 } & & \\ \text { Bristol Myers Squibb } & \text { BMS-986089 } & \text { Anti-MSTN adnectin } & 116 \\ \text { Roche/Cenentech } & \text { R07239361 } & & \\ \text { Scholar Rock } & \text { SRK-015 } & \text { Anti-propeptide monoclonal antibody } & 47,117 \\ \text { Novartis } & \text { Bimagrumab } & \text { Anti-receptor monoclonal antibody } & 48,99,118 \\ \text { Acceleron } & \text { ACE-031 } & \text { Decoy receptor } & 111 \\ & \text { ACE-083 } & \text { Follistatin/Fc fusion } & 64\end{array}$

When not bound to inhibitory proteins, MSTN signals using a two-component receptor system as is typical for most members of the TGF- $\beta$ superfamily. Receptor binding studies showed that MSTN is capable of binding to the two activin type II receptors, ACVR2 and ACVR2B (also called ActRIIA and ActRIIB) (12). Because MSTN was shown to bind ACVR2B with higher affinity than ACVR2, the initial assumption was that ACVR2B perhaps plays a more important role than ACVR2 in mediating MSTN signaling in vivo. Indeed, transgenic overexpression of a dominant-negative form of ACVR2B lacking the cytoplasmic signaling domain in skeletal muscle phenocopied Mstn-null mice in terms of increased muscle mass (12). Moreover, a decoy receptor consisting of the extracellular domain of ACVR2B fused to an immunoglobulin Fc domain was shown to be a potent inhibitor of MSTN both in vitro and in vivo (33); in fact, systemic administration of this ACVR2B/Fc decoy receptor to adult mice is capable of inducing over $50 \%$ muscle growth in just 2 weeks. Genetic studies have shown, however, that both ACVR2 and ACVR2B play important roles in regulating MSTN activity in vivo. In particular, analysis of mice carrying deletion mutations in Acvr2 and $A c v r 2 b$ showed that loss of either receptor can lead to increased muscle mass and that the two receptors are partially functionally redundant with each other (33). Moreover, studies using floxed alleles of these genes showed that targeting the receptors specifically in skeletal myofibers is sufficient to increase muscle growth (34). These studies, in conjunction with the analysis of the effect of MSTN inhibition in mice carrying mutations in key genes involved in satellite cell function, as well as direct analysis of satellite cells in mice in which MSTN was blocked pharmacologically, demonstrated unequivocally that the myofibers themselves are direct targets for MSTN signaling in the regulation of muscle growth (35).

Binding of MSTN to the activin type II receptors then leads to engagement of the type I receptors, ALK4 and ALK5 (36). Recent genetic studies have confirmed that both ALK4 and ALK5 play important roles in regulating muscle mass (34). Specifically, targeting either of these type I receptors specifically in myofibers can lead to small increases in muscle mass, but targeting both simultaneously can lead to dramatic muscle growth, demonstrating that these two receptors are partially functionally redundant with each other. Moreover, studies analyzing the effect of targeting each combination of one type II with one type I receptor showed that all four possible combinations of type II and type I receptors are used in vivo in the control of muscle growth. A final receptor component that has been implicated in MSTN signaling is cripto (cryptic family protein $1 b$, encoded by $C f c 1 b$ ), which is known to serve as a coreceptor for certain ligands and to antagonize the activity of other ligands (reviewed in ref. 37). One study reported that cripto is required for MSTN signaling in cultured myoblasts (38), but another study reported that cripto expressed in satellite cells during muscle regeneration inhibits MSTN signaling (39). Genetic studies targeting $C f c 1 b$ in myofibers showed that cripto may play a minor role in regulating muscle growth (34).

In addition to confirming the key roles that these regulatory components play in vivo, genetic and pharmacological studies targeting various components have also provided unexpected insights into the complexity of this signaling system. In particular, the ACVR2B/Fc decoy receptor was found to be capable of inducing muscle growth not only in wild-type mice but also in $\mathrm{Mstn}^{-/-}$mice (33), and transgenic overexpression of FST in $\mathrm{Mstn}^{-/}$mice was shown to cause yet another doubling, i.e., an overall quadrupling, of muscle mass (26). These results implied the existence of at least one other TGF- $\beta$ family member whose function is partially redundant with that of MSTN. Subsequent work has identified activin A as at least one key ligand that cooperates with MSTN to limit muscle growth (24, $40,41)$, although it remains possible that other members of the TGF- $\beta$ superfamily may also play a role. In this respect, a second unexpected finding was the discrepancy in phenotype that was observed between targeting of the two type II receptors, Acvr2 and $A c v r 2 b$, and targeting of the two type I receptors, Alk4 and Alk5, in myofibers (34). In particular, many of the mice in which Alk4 and Alk5 were targeted exhibited significantly more muscle growth than mice in which Acvr2 and Acvr $2 b$ were targeted, raising the possibility that other ligands or receptors might also be involved.

\section{Clinical trials testing MSTN inhibitors}

The elucidation of key components of the MSTN regulatory system and the results of preclinical studies examining the effects of targeting MSTN in mouse models of human diseases have fueled an extensive effort to develop MSTN inhibitors for clinical use (Table 1). All of the MSTN inhibitors tested in clinical trials to date have been biologics and have fallen into two general classes. One class (MYO-029, domagrozumab, LY2495655, REGN1033, AMG-745/PINTA-745, BMS986089/RO7239361, SRK-015) comprises those molecules that are relatively specific for MSTN, although some exhibit cross-reactivity to the MSTN-related protein GDF-11. The second class (bimagrumab, ACE-031, ACE-083) comprises those molecules that have a broader range of ligand specificity and are capable of blocking not just MSTN and GDF-11 but also activin A. Collectively, these biologics have been tested in clinical trials for a wide range of indications (Table 2).

Based on the data published from these clinical trials, several general conclusions seem evident. First, targeting the MSTN signaling pathway in humans did lead to increased muscling, with the magnitude of the effect generally being higher with biologics capable of targeting more than just MSTN. Many of the trials reported increased thigh muscle volume by MRI and increased lean body mass by dual-energy $\mathrm{x}$-ray absorptiometry (DXA), with effects in 


\section{Table 2. Clinical trials testing MSTN inhibitors}

\begin{tabular}{|c|c|c|c|c|}
\hline Drug & Indication & Phase & ID & Ref. \\
\hline MYO-029 & $\begin{array}{l}\text { Becker muscular dystrophy } \\
\text { Facioscapulohumeral muscular dystrophy } \\
\text { Limb-girdle muscular dystrophy }\end{array}$ & Phase I/II & NCT00104078 & 51 \\
\hline Domagrozumab & Duchenne muscular dystrophy & Phase II & $\begin{array}{l}\text { NCT02310763 } \\
\text { NCT02907619 }\end{array}$ & $\begin{array}{l}52,53 \\
52,53\end{array}$ \\
\hline R07239361 & Duchenne muscular dystrophy & Phase II & NCT03039686 & - \\
\hline ACE-031 & Duchenne muscular dystrophy & Phase II & $\begin{array}{l}\text { NCT01099761 } \\
\text { NCT01239758 }\end{array}$ & $\begin{array}{l}79 \\
79\end{array}$ \\
\hline Domagrozumab & Limb-girdle muscular dystrophy 21 & Phase I/II & NCT02841267 & - \\
\hline Bimagrumab & Sporadic inclusion body myositis & $\begin{array}{l}\text { Phase II } \\
\text { Phase II } \\
\text { Phase II/III } \\
\text { Phase III }\end{array}$ & $\begin{array}{l}\text { NCT01423110 } \\
\text { NCT01925209 } \\
\text { NCT02250443 } \\
\text { NCT02573467 }\end{array}$ & $\begin{array}{l}119 \\
54 \\
55 \\
-\end{array}$ \\
\hline SRK-015 & Spinal muscular atrophy & Phase II & NCT03921528 & - \\
\hline LY2495655 & Pancreatic cancer & Phase II & NCT01505530 & 56 \\
\hline Bimagrumab & Lung or pancreatic cancer & Phase II & NCT01433263 & - \\
\hline Bimagrumab & Chronic obstructive pulmonary disease & Phase II & NCT01669174 & 57 \\
\hline PINTA-745 & End-stage renal disease & Phase I/II & NCT01958970 & - \\
\hline Bimagrumab & Sarcopenia & $\begin{array}{l}\text { Phase II } \\
\text { Phase II } \\
\text { Phase II }\end{array}$ & $\begin{array}{r}\text { NCT01601600 } \\
\text { NCT02333331 } \\
\text { NCT02468674 }\end{array}$ & $\begin{array}{l}58 \\
66 \\
-\end{array}$ \\
\hline LY2495655 & Hip replacement & Phase II & NCT01369511 & 69 \\
\hline LY2495655 & Muscle weakness following falls & Phase II & NCT01604408 & 59 \\
\hline Bimagrumab & Hip fracture surgery & Phase II & NCT02152761 & - \\
\hline REGN1033 & Sarcopenia & Phase II & NCT01963598 & - \\
\hline Bimagrumab & Obese patients with type 2 diabetes & Phase II & NCT03005288 & 65 \\
\hline
\end{tabular}

the range of $3 \%$ to $9 \%$ depending on the specific trial. These effects, though significant, were substantially lower than those seen in mice, in which muscle mass increases in the range of $10 \%$ to $30 \%$ have been reported with biologics relatively specific for MSTN (42-47), and even greater increases have been achieved in mice with biologics capable of blocking both MSTN and activin A. Specifically, bimagrumab, which targets receptors used by both MSTN and activin A, showed increases of $25 \%$ to $50 \%$ in mice (48), and the ACVR2B/Fc decoy receptor, which can act as a ligand trap for both MSTN and activin A, showed increases of over $50 \%$ in mice (33).

In this respect, the effects seen in the human trials also seemed to be generally higher with biologics that had a broader specificity in terms of ligands being targeted. Treating patients with bimagrumab or ACE-031 led to increases in thigh muscle volume by $5 \%$ to $9 \%$, compared with increases of $3 \%$ to $5 \%$ seen upon treatment with agents more specific for MSTN; in fact, in healthy adults, increases of over $5 \%$ were seen 4 weeks after just a single injection of either bimagrumab (49) or ACE-031 (50). The larger effects on muscle mass seen with agents capable of targeting both MSTN and activin A were consistent not only with mouse studies showing that both proteins are involved in regulating muscle growth but also with the relative circulating levels of these proteins in humans versus mice. In particular, circulating levels of MSTN and activin A are approximately 7- to 8-fold lower and 3- to 4-fold higher, respectively, in humans compared with mice, suggesting that activin A may play a more prominent role in humans compared with mice (40).
Second, the response to MSTN inhibition in terms of functional improvements has been inconsistent and seemed to vary not only with the specific biologic being tested but also with the indication. In most of the trials, increased muscle mass did not translate into clinically meaningful improvements with respect to measurements of strength, such as hand grip or knee extension, or other functional assessments, such as 6-minute walk distance, stair climbing time, other physical performance tests, or self-reported physical function. This failure to achieve clinical functional benefit was seen in all of the trials that focused on patients with muscular dystrophy (51-53), Novartis's trial of bimagrumab in patients with sporadic inclusion body myositis $(54,55)$, and trials testing MSTN inhibitors in patients with cachexia from cancer (56), chronic obstructive pulmonary disease (57), and end-stage renal disease. Two of the trials that did show clear functional improvements were in the elderly population. In one trial, bimagrumab treatment of individuals aged 65 or older with sarcopenia led to increased grip strength and, in a subset of individuals, increased gait speed and 6-minute walk distance as well (58). In another trial, LY2495655 treatment of individuals aged 75 or older who had fallen led to significant improvements in stair climbing, time to rise from a chair, and gait speed (59).

Almost certainly, a major reason for the inconsistency in functional improvements is the relatively small magnitude of muscle mass increases seen in humans compared with mice, but other issues need to be considered as well. One issue that has been raised is that studies in mice have shown that muscle quality may be suboptimal upon MSTN inhibition, for example with respect to specific force (60-62) or fatigability (63). Numerous studies, however, have documented increases in total force and improved outcomes in mice treated with MSTN inhibitors, including with many of the biologics that were tested in clinical trials $(43-45,47,48,64)$. A second issue is that although actual muscle strength was used as a metric in some of the trials, other functional parameters may not directly track with muscle mass or strength, and a major challenge for many indications is defining appropriate functional endpoints that may reflect the beneficial effects of MSTN inhibition.

Third, targeting the MSTN signaling pathway had consistent effects in reducing fat mass. This effect was clearly seen in Novartis's trial of bimagrumab in obese individuals with type 2 diabetes (65), which reported perhaps the most promising results to date. Treatment with bimagrumab led to highly significant $(P<0.001)$ decreases in total body fat mass by $20 \%$, increases in lean body mass by $4.4 \%$, and decreases in waist circumference by $9.5 \mathrm{~cm}$. This effect of MSTN inhibition on reducing fat mass was also observed in many of the other clinical trials and clinical studies, not only with bimagrumab $(49,55,57,58,66-68)$ but also with LY2495655 (59, 69), AMG-745 (70), and ACE-031 (50). These findings were consis- 
tent with mouse studies demonstrating that targeting MSTN signaling can significantly reduce fat accumulation, which was initially reported in mice completely lacking MSTN (71) and subsequently shown in mice treated with MSTN inhibitors (72-74). Targeting this pathway in mice was also shown to have beneficial effects on glucose metabolism in both genetic (71) and diet-induced (72) models of obesity and type 2 diabetes. Indeed, beneficial metabolic effects of bimagrumab were also clearly demonstrated in Novartis's trial in obese individuals with type 2 diabetes, in which drug treatment lowered glycated hemoglobin (HbA1c) by 0.76 percentage points compared with placebo (65). Moreover, in another clinical study with bimagrumab, reductions in fat mass, improvements in insulin sensitivity, and lowering of HbA1c were even apparent 10 weeks after just a single dose (68).

The physiological mechanisms underlying the metabolic benefits and reduction in fat mass seen in response to systemic inhibition of this pathway, such as with the ACVR2B/Fc decoy receptor, are likely complex. The anabolic effects on muscle are clearly an important component of these effects on fat mass and glucose metabolism. Genetic studies in mice have shown that simultaneously targeting the two type II receptors, ACVR2 and ACVR2B, specifically in myofibers can cause not only dramatic increases in muscle mass but also significant reductions in overall body fat and improvements in glucose metabolism, demonstrating unequivocally that blocking signaling in muscle is sufficient to generate these effects (34). Indeed, the anabolic effects in muscle leading to increased insulin sensitivity, increased glucose uptake, and increased glycogen storage $(73,75,76)$ almost certainly play a major role in the systemic metabolic benefits seen in mice treated with MSTN inhibitors. It is also possible, however, that inhibition of signaling to other cell types may play a role as well. For example, MSTN inhibition in mice leads to increased basal metabolic rate (73), which may result at least in part from loss of MSTN signaling to brown preadipocytes. In particular, MSTN is capable of blocking brown adipocyte differentiation in vitro $(77,78)$, and inhibition of this pathway in mice has been shown to increase the amount of brown adipose tissue, leading to increased energy expenditure (78).

\section{Future possibilities}

Although the results of many of the early clinical trials targeting MSTN had been rather disappointing, more promising results have been reported in some of the newer trials, and further work to develop strategies to target the MSTN signaling pathway for clinical applications seems warranted. In developing such strategies, two general issues need to be considered: the drug and the clinical indication.

The first issue is the drug itself, and a major question in this regard is the optimal specificity of the drug with respect to the ligands being targeted. Studies in mice have shown that targeting MSTN alone is sufficient to induce muscle growth but that targeting MSTN along with other TGF- $\beta$ family members, most notably activin A, can have a much greater effect $(24,26,33,40,41)$, and this seems to have been borne out in the clinical trials as well. Of course, the broader ligand specificity of molecules like ACVR2B/ Fc expands the possible risks for undesired effects outside of skeletal muscle, and indeed, adverse effects, namely epistaxis and telangiectasias, were observed in Acceleron's trial using their version of this decoy receptor in patients with Duchenne muscular dystrophy (79). These adverse effects were attributed to the ability of ACVR2B/Fc to block the TGF- $\beta$ family members BMP9 (GDF-2) and BMP-10. Both BMP-9 and BMP-10 are known to play important roles in vascular development (reviewed in ref. 80 ), and BMP9 mutations have been identified in individuals with clinical features of hereditary hemorrhagic telangiectasia (81). Why these adverse effects have not yet been observed in any of the trials using bimagrumab is not clear, but it is important to keep in mind that because ACVR2B/Fc acts as a ligand trap, it is capable of inhibiting any TGF- $\beta$ family member that can bind ACVR2B, whereas bimagrumab will only inhibit ligands that actually signal through the activin type II receptors in a given cell type. Hence, it is possible, for example, that ACVR2B/Fc is capable of binding and inhibiting a ligand like BMP-9 or BMP10 , even if actual signaling by these ligands is mediated partly or entirely by a different receptor in the relevant cell types. Indeed, one study reported that another type II receptor, BMPR2, may be critical in mediating a subset of BMP-9 responses (82).

Given the likelihood that agents with a broader range of ligand specificity will be more potent in terms of inducing muscle growth than MSTN-specific agents, the question is how to tap into their anabolic potential while simultaneously avoiding possible adverse effects on other tissues and cell types. One strategy could be to start with a molecule specific for MSTN and then add in other agents capable of blocking other specific TGF- $\beta$ family members, like activin A. Indeed, a combination approach of simultaneously giving two monoclonal antibodies, one directed against MSTN and the other directed against activin A, was shown to be significantly more effective than either one alone in mice (40). An alternative strategy could be to start with a molecule with a broad range of ligand specificity, such as ACVR2B/Fc, and then introduce modifications to "dial out" undesired interactions with certain ligands, like BMP-9 and/or BMP-10. A similar approach could also be used with a biologic like FST. FST contains three follistatin domains that are involved in mediating binding to various TGF- $\beta$ family members, including MSTN, GDF- 11 , activin A, and certain BMPs. Follistatin domains are also present in FSTL-3, and the demonstration that individual follistatin domains are preferentially involved in binding of these molecules to different TGF- $\beta$ family members has suggested the possibility that molecules with varying specificity for different ligands could be engineered using novel combinations of these domains $(83,84)$.

Of course, even with a therapeutic approach that is highly specific for MSTN and activin A, it is likely that inhibition of these molecules will have effects outside of skeletal muscle. Receptors for these molecules are expressed by many cells and tissues throughout the body, and both MSTN and activin A circulate in the blood. Hence, these ligands could, in principle, signal to any cells that express their receptors, although activation of latency would be an additional requirement in the case of MSTN. In this respect, it is known, for example, that systemic administration of ACVR2B/ Fc to mice is capable of not only causing dramatic skeletal muscle growth (33), suppression of fat accumulation, and improvements in glucose metabolism $(72,74)$ but also having other effects, including increases in bone mineral density $(74,85-88)$ and protection against heart injury (89), all of which likely reflect inhibition of 
MSTN and/or activin A signaling. Blocking activin signaling to gonadotrophs in the pituitary gland can also lead to lowering of FSH secretion (90), which was seen in postmenopausal women treated with bimagrumab (91). Lowering FSH levels could not only have consequences for reproductive function and estrogen production but also impact extragonadal functions of FSH, such as regulation of bone (92) and adipose tissue (93). On the one hand, many of these effects of blocking MSTN and activin A signaling may be beneficial for certain indications, such as aging as well as other conditions characterized by comorbid muscle and bone loss (47, $88,94,95)$. On the other hand, however, not being able to separate these biological effects may be suboptimal for many clinical indications. Clearly, the development of methods to deliver biologics specifically to skeletal muscle would potentially get around these off-muscle effects and would also open up numerous possibilities in terms of specific components of this regulatory system to target. For example, targeting the two type I receptors, ALK4 and ALK5, seems to have a much greater effect on muscle mass than targeting the two type II receptors, ACVR2 and ACVR2B, at least in mice (34). If this is also true in humans, the expectation would be that targeting the type I receptors could lead to much more substantial effects on muscle mass than the 3\%-9\% increases that have been observed in the trials to date, which might increase the likelihood of seeing clinically meaningful functional benefits.

The second major issue is the clinical indication, and the question is which indications are likely to be most responsive to MSTN inhibition. One reasonable approach has been to attempt to target those disease states in which overactivity of this signaling pathway may contribute to the overall pathology in terms of muscle loss. Several of the Novartis trials, in fact, tested bimagrumab in two such conditions: sporadic inclusion body myositis, in which Smad signaling was found to be increased dramatically in skeletal muscle (54), and chronic obstructive pulmonary disease, in which MSTN was found to be upregulated in muscle $(96,97)$. Both of these trials, however, failed to demonstrate clinical benefit from drug treatment. Another example is that overexpression of MSTN can induce cachexia in mice (14), and increased pathway activity has been implicated in cancer cachexia in humans, with activin A levels reported to be higher in cachectic than in non-cachectic cancer patients (98). Blocking this signaling pathway has been shown not only to preserve muscle mass in cachexia models (99-101) but even to prolong survival of these mice (99, 101). Increased survival, however, was not achieved in Eli Lilly's trial of LY2495655 in patients with pancreatic cancer, the caveat being that their drug does not inhibit activin A. Another example of a condition in which upregulation of signaling may play a causative role is muscle atrophy induced by glucocorticoids. MSTN expression has been shown to be upregulated by glucocorticoids (102-104), which is mediated by glucocorticoid response elements present in the MSTN promoter $(103,105)$, and inhibition of MSTN has been shown to block glucocorticoid-induced muscle atrophy in mice $(45,48,106)$. Although MSTN inhibition has not been tested specifically to counter the effects of glucocorticoids in humans, many of the patients in the muscular dystrophy trials were taking steroids as part of their routine clinical care, and as discussed above, none of those trials demonstrated clinical benefit. Finally, circulating levels of activin A have been shown to correlate with worsening heart failure (89), and circulating levels of GDF-11 have been shown to correlate with poorer outcome in individuals undergoing treatment for aortic stenosis (107). Although agents targeting this pathway have not yet been tested in patients with heart disease, blocking signaling by these ligands was shown to have a protective effect in mice (89). Moreover, an intriguing study in mice suggested that MSTN made by the heart may be a key mediator of skeletal muscle wasting, or cardiac cachexia (108).

The converse to all of this could be that conditions in which the MSTN pathway is already downregulated as a result of the disease process may be less responsive to MSTN inhibition. Indeed, the MSTN pathway has been shown to be downregulated in patients with various neuromuscular diseases, including Duchenne muscular dystrophy $(109,110)$. One difficulty in interpreting these findings in the context of clinical trial failures, however, is that at least in the case of MSTN, lower circulating levels may simply reflect the lower amount of functional skeletal muscle tissue in these disease states, given that myofibers are the predominant source of circulating MSTN protein (34).

As pointed out above, two trials in the elderly population showed improvement in functional measurements as a result of drug treatment. This is perhaps noteworthy because two theoretical concerns have been raised regarding this type of therapeutic approach to combat age-related sarcopenia. One concern has been that depletion of satellite cells during aging may render aged muscle unresponsive to MSTN inhibition. Studies in mice, however, have clearly demonstrated that the myofibers themselves are direct targets for MSTN signaling and that blocking signaling in myofibers is sufficient to induce hypertrophy without satellite cell involvement $(34,35)$. A second concern has been that the preferential loss of type II fibers during aging might also make this therapeutic approach ineffective. Analysis of mice treated with ACVR2B/Fc, however, has clearly documented that both type I and type II fibers undergo hypertrophy when this signaling pathway is blocked (111). Although the results of these two trials suggest that the elderly population may be responsive to this therapeutic strategy, developing drugs to combat age-related sarcopenia is particularly challenging both in terms of recruiting subjects for clinical trials and in terms of defining suitable outcome measures to reach FDA approval.

With respect to other indications that may benefit from this approach, preliminary unpublished reports suggest that Scholar Rock's monoclonal SRK-015 may have beneficial effects in patients with spinal muscular atrophy (SMA). If these promising results continue to hold up, it will be important to determine whether these beneficial effects reflect SMA being a particularly good indication from the perspective of responsiveness to pathway inhibition or whether they reflect the biological properties of SRK-015 with respect to its mechanism of action in blocking activation of MSTN from its latent state. Perhaps noteworthy in this regard is that one study reported massively increased levels of circulating GDF-11 in patients with SMA (110). This finding raises the possibility that increased GDF-11 signaling may be contributing to pathogenesis in this disorder and that agents capable of targeting both GDF-11 and MSTN may have greater efficacy in SMA than SRK-015, which is highly specific for MSTN. 
Nearly all of the trials to date have focused on possible benefits of increased muscle mass in terms of functional parameters, such as strength, walking distance, stair climbing time, and gait speed. Much less attention has been focused on the metabolic benefits of blocking this signaling pathway, and the promising results of the trial by Novartis testing bimagrumab in obese individuals with type 2 diabetes certainly support widening the focus in terms of indications. Studies in mice have demonstrated clearly that blocking signaling by ACVR2 and ACVR2B just in skeletal myofibers not only can induce significant muscle growth but also can reduce total body fat content and improve glucose metabolism (34). This is perhaps not surprising given that skeletal muscle comprises about a third of overall body weight and is the tissue most responsible for taking up glucose from the blood. In this respect, I have speculated previously that one of the reasons that the MSTN regulatory system has evolved to be so complex, with multiple extracellular binding proteins and mechanisms for regulating MSTN latency, is that these regulatory mechanisms allow for local control of muscle growth in response to specific stimuli while at the same time allowing for systemic control of the overall balance between fat and muscle throughout the body $(112,113)$. Indeed, it is perhaps this fundamental role of MSTN in regulating the overall metabolic homeostatic balance between fat storage and muscle growth that is responsible for the extraordinary degree to which MSTN has been conserved through evolution. In this respect, the benefits seen in the trial testing bimagrumab in obesity and type 2 diabetes certainly suggest that skeletal muscle as a metabolic tissue may be at least as important as skeletal muscle as a contractile organ in considering possible health benefits of MSTN inhibition.

\section{Acknowledgments}

Work in the author's laboratory is supported by NIH grants R01AR060636 and R01AG052962.

Address correspondence to: Se-Jin Lee, The Jackson Laboratory, 10 Discovery Drive, Farmington, Connecticut 06032, USA; Phone: 860.837.2183; Email: Se-Jin.Lee@jax.org.
1. McPherron AC, et al. Regulation of skeletal muscle mass in mice by a new TGF- $\beta$ superfamily member. Nature. 1997;387(6628):83-90.

2. McPherron AC, Lee SJ. Double muscling in cattle due to mutations in the myostatin gene. Proc Natl Acad Sci U S A. 1997;94(23):12457-12461.

3. Grobet L, et al. A deletion in the bovine myostatin gene causes the double-muscled phenotype in cattle. Nat Genet. 1997;17(1):71-74.

4. Kambadur R, et al. Mutations in myostatin (GDF8) in double-muscled Belgian Blue and Piedmontese cattle. Genome Res. 1997;7(9):910-916.

5. Clop A, et al. A mutation creating a potential illegitimate microRNA target site in the myostatin gene affects muscularity in sheep. Nat Genet. 2006;38(7):813-818.

6. Mosher DS, et al. A mutation in the myostatin gene increases muscle mass and enhances racing performance in heterozygote dogs. PLoS Genet. 2007;3(5):779-786.

7. Lv Q, et al. Efficient generation of myostatin gene mutated rabbit by CRISPR/Cas9. Sci Rep. 2016;6:25029.

8. Gu H, et al. Establishment and phenotypic analysis of an Mstn knockout rat. Biochem Biophys Res Commun. 2016;477(1):115-122.

9. Wang K, et al. CRISPR/Cas9-mediated knockout of myostatin in Chinese indigenous Erhualian pigs. Transgenic Res. 2017;26(6):799-805.

10. He Z, et al. Use of CRISPR/Cas9 technology efficiently targetted goat myostatin through zygotes microinjection resulting in double-muscled phenotype in goats. Biosci Rep. 2018;38(6):BSR20180742.

11. Schuelke M, et al. Myostatin mutation associated with gross muscle hypertrophy in a child. $N$ Engl J Med. 2004;350(26):2682-2688.

12. Lee SJ, McPherron AC. Regulation of myostatin activity and muscle growth. Proc Natl Acad Sci U S A. 2001;98(16):9306-9311.

13. Thies RS, et al. GDF-8 propeptide binds to GDF-8 and antagonizes biological activity by inhib- iting GDF-8 receptor binding. Growth Factors. 2001;18(4):251-259.

14. Zimmers TA, et al. Induction of cachexia in mice by systemically administered myostatin. Science. 2002;296(5572):1486-1488.

15 . Hill JJ, et al. The myostatin propeptide and the follistatin-related gene are inhibitory binding proteins of myostatin in normal serum. J Biol Chem. 2002;277(43):40735-40741.

16. Wolfman NM, et al. Activation of latent myostatin by the BMP-1/tolloid family of metalloproteinases. Proc Natl Acad Sci US A. 2003;100(26):15842-15846.

17. Lee S-J. Genetic analysis of the role of proteolysis in the activation of latent myostatin. PLoS One. 2008;3(2):e1628.

18. Nakamura T, et al. Activin-binding protein from rat ovary is follistatin. Science. 1990;247(4944):836-838.

19. Yamashita H, et al. Osteogenic protein-1 binds to activin type II receptors and induces certain activin-like effects. J Cell Biol. 1995;130(1):217-226.

20. Fainsod A, et al. The dorsalizing and neural inducing gene follistatin is an antagonist of BMP4. Mech Dev. 1997;63(1):39-50.

21. Iemura S, et al. Direct binding of follistatin to a complex of bone-morphogenetic protein and its receptor inhibits ventral and epidermal cell fates in early Xenopus embryo. Proc Natl Acad Sci U S A. 1998;95(16):9337-9342.

22. Gamer LW, et al. A novel BMP expressed in developing mouse limb, spinal cord, and tail bud is a potent mesoderm inducer in Xenopus embryos. Dev Biol. 1999;208(1):222-232.

23. Matzuk MM, et al. Multiple defects and perinatal death in mice deficient in follistatin. Nature. 1995;374(6520):360-363.

24. Lee S-J, et al. Regulation of muscle mass by follistatin and activins. Mol Endocrinol. 2010;24(10):1998-2008.

25. Cash JN, et al. Structure of myostatin $\cdot$ follistatin-like 3: N-terminal domains of follistatin-type molecules exhibit alternate modes of binding. $J$ Biol Chem. 2012;287(2):1043-1053.

26. Lee SJ. Quadrupling muscle mass in mice by targeting TGF- $\beta$ signaling pathways. PloS One. 2007;2(8):e789.

27. Haidet AM, et al. Long-term enhancement of skeletal muscle mass and strength by single gene administration of myostatin inhibitors. Proc Natl Acad SciU S A. 2008;105(11):4318-4322.

28. Mukherjee A, et al. FSTL3 deletion reveals roles for TGF-beta family ligands in glucose and fat homeostasis in adults. Proc Natl Acad Sci U S A. 2007;104(4):1348-1353.

29. Hill JJ, et al. Regulation of myostatin in vivo by growth and differentiation factor-associated serum protein-1: a novel protein with protease inhibitor and follistatin domains. Mol Endocrinol. 2003;17(6):1144-1154.

30. Trexler M, et al. Distinct expression pattern of two related human proteins containing multiple types of protease-inhibitory modules. Biol Chem 2002;383(1):223-228.

31. Lee YS, Lee SJ. Regulation of GDF-11 and myostatin activity by GASP-1 and GASP-2. Proc Natl Acad Sci U S A. 2013;110(39):E3713-E3722.

32. Kondás K, et al. Both WFIKKN1 and WFIKKN2 have high affinity for growth and differentiation factors 8 and 11. J Biol Chem. 2008;283(35):23677-23684.

33. Lee SJ, et al. Regulation of muscle growth by multiple ligands signaling through activin type II receptors. Proc Natl Acad Sci U S A. 2005;102(50):18117-18122.

34. Lee SJ, et al. Functional redundancy of type I and type II receptors in the regulation of skeletal muscle growth by myostatin and activin A. Proc Natl Acad Sci U S A. 2020;117(49):30907-30917.

35. Lee SJ, et al. Role of satellite cells versus myofibers in muscle hypertrophy induced by inhibition of the myostatin/activin signaling pathway. Proc Natl Acad Sci U S A. 2012;109(35):E2353-E2360 36. Rebbapragada A, et al. Myostatin signals through 
a transforming growth factor beta-like signaling pathway to block adipogenesis. Mol Cell Biol. 2003;23(20):7230-7242.

37. Nagaoka T, et al. An evolving web of signaling networks regulated by Cripto-1. Growth Factors. 2012;30(1):13-21.

38. Kemaladewi DU, et al. Cell-type specific regulation of myostatin signaling. FASEB J. 2012;26(4):1462-1472.

39. Guardiola O, et al. Cripto regulates skeletal muscle regeneration and modulates satellite cell determination by antagonizing myostatin. Proc Natl Acad Sci U S A. 2012;109(47):E3231-E3240.

40. Latres E, et al. Activin A more prominently regulates muscle mass in primates than does GDF8. Nat Commun. 2017;8:15153.

41. Chen JL, et al. Specific targeting of TGF- $\beta$ family ligands demonstrates distinct roles in the regulation of muscle mass in health and disease. Proc Natl Acad Sci U S A. 2017;114(26):E5266-E5275.

42. Apgar JR, et al. Beyond CDR-grafting: structure-guided humanization of framework and CDR regions of an anti-myostatin antibody. MAbs. 2016;8(7):1302-1318.

43. St Andre M, et al. A mouse anti-myostatin antibody increases muscle mass and improves muscle strength and contractility in the $\mathrm{mdx}$ mouse model of Duchenne muscular dystrophy and its humanized equivalent, domagrozumab (PF-06252616), increases muscle volume in cynomolgus monkeys. Skelet Muscle. 2017;7(1):25.

44. Smith RC, et al. Myostatin neutralization results in preservation of muscle mass and strength in preclinical models of tumor-induced muscle wasting. Mol Cancer Ther. 2015;14(7):1661-1670.

45. Latres E, et al. Myostatin blockade with a fully human monoclonal antibody induces muscle hypertrophy and reverses muscle atrophy in young and aged mice. Skelet Muscle. 2015;5:34.

46. Zhang L, et al. Pharmacological inhibition of myostatin suppresses systemic inflammation and muscle atrophy in mice with chronic kidney disease. FASEB J. 2011;25(5):1653-1663.

47. Long KK, et al. Specific inhibition of myostatin activation is beneficial in mouse models of SMA therapy. Hum Mol Genet. 2019;28(7):1076-1089.

48. Lach-Trifilieff E, et al. An antibody blocking activin type II receptors induces strong skeletal muscle hypertrophy and protects from atrophy. Mol Cell Biol. 2014;34(4):606-618.

49. Rooks D, et al. Safety and pharmacokinetics of bimagrumab in healthy older and obese adults with body composition changes in the older cohort. J Cachexia Sarcopenia Muscle. 2020;11(6):1525-1534.

50. Attie KM, et al. A single ascending-dose study of muscle regulator ACE-031 in healthy volunteers. Muscle Nerve. 2013;47(3):416-423.

51. Wagner KR, et al. A phase I/II trial of MYO-029 in adult subjects with muscular dystrophy. Ann Neurol. 2008;63(5):561-571.

52. Wagner KR, et al. Randomized phase 2 trial and open-label extension of domagrozumab in Duchenne muscular dystrophy. Neuromuscul Disord. 2020;30(6):492-502.

53. Wagner KR, et al. Corrigendum to "Randomized phase 2 trial and open-label extension of domagrozumab in Duchenne muscular dystro- phy" [Neuromuscul Disord 30(6);2020:492-502]. Neuromuscul Disord. 2021;31(2):167-168.

54. Hanna MG, et al. Safety and efficacy of intravenous bimagrumab in inclusion body myositis (RESILIENT): a randomised, double-blind, placebo-controlled phase $2 \mathrm{~b}$ trial. Lancet Neurol. 2019;18(9):834-844.

55. Sivakumar K, et al. Long-term safety and tolerability of bimagrumab (BYM338) in sporadic inclusion body myositis. Neurology. 2020;95(14):e1971-e1978.

56. Golan T, et al. LY2495655, an antimyostatin antibody, in pancreatic cancer: a randomized, phase 2 trial. JCachexia Sarcopenia Muscle. 2018;9(5):871-879.

57. Polkey MI, et al. Activin type II receptor blockade for treatment of muscle depletion in chronic obstructive pulmonary disease. A randomized trial. Am J Respir Crit Care Med.2019;199(3):313-320.

58. Rooks D, et al. Treatment of sarcopenia with bimagrumab: results from a phase II, randomized, controlled, proof-of-concept study. J Am Geriatr Soc. 2017;65(9):1988-1995.

59. Becker C, et al. Myostatin antibody (LY2495655) in older weak fallers: a proof-of-concept, randomised, phase 2 trial. Lancet Diabetes Endocrinol. 2015;3(12):948-957.

60. Mendias CL, et al. Contractile properties of EDL and soleus muscles of myostatin-deficient mice. $J$ Appl Physiol (1985). 2006;101(3):898-905.

61. Amthor $\mathrm{H}$, et al. Lack of myostatin results in excessive muscle growth but impaired force generation. Proc Natl Acad Sci U S A. 2007;104(6):1835-1840.

62. Giannesini B, et al. Lack of myostatin impairs mechanical performance and ATP cost of contraction in exercising mouse gastrocnemius muscle in vivo. Am J Physiol Endocrinol Metab. 2013;305(1):E33-E40.

63. Relizani K, et al. Blockade of ActRIIB signaling triggers muscle fatigability and metabolic myopathy. Mol Ther. 2014;22(8):1423-1433.

64. Pearsall RS, et al. Follistatin-based ligand trap ACE-083 induces localized hypertrophy of skeletal muscle with functional improvement in models of neuromuscular disease. Sci Rep. 2019;9(1):11392.

65. Heymsfield SB, et al. Effect of bimagrumab vs placebo on body fat mass among adults with type 2 diabetes and obesity: a phase 2 randomized clinical trial. JAMA Netw Open. 2021;4(1):e2033457.

66. Rooks D, et al. Bimagrumab vs optimized standard of care for treatment of sarcopenia in community-dwelling older adults: a randomized clinical trial. JAMA Netw Open. 2020;3(10):e2020836.

67. Rooks DS, et al. Effect of bimagrumab on thigh muscle volume and composition in men with casting-induced atrophy. JCachexia Sarcopenia Muscle. 2017;8(5):727-734.

68. Garito T, et al. Bimagrumab improves body composition and insulin sensitivity in insulin-resistant individuals. Diabetes Obes Metab. 2018;20(1):94-102.

69. Woodhouse $\mathrm{L}$, et al. A phase 2 randomized study investigating the efficacy and safety of myostatin antibody LY2495655 versus placebo in patients undergoing elective total hip arthroplasty. J Frailty Aging. 2016;5(1):62-70.

70. Padhi D, et al. Pharmacological inhibition of myostatin and changes in lean body mass and lower extremity muscle size in patients receiving androgen deprivation therapy for prostate cancer. J Clin Endocrinol Metab. 2014;99(10):E1967-E1975.

71. McPherron AC, Lee SJ. Suppression of body fat accumulation in myostatin-deficient mice. JClin Invest. 2002;109(5):595-601.

72. Akpan I, et al. The effects of a soluble activin type IIB receptor on obesity and insulin sensitivity. Int JObes (Lond). 2009;33(11):1265-1273.

73. LeBrasseur NK, et al. Myostatin inhibition enhances the effects of exercise on performance and metabolic outcomes in aged mice. J Gerontol A Biol Sci Med Sci. 2009;64(9):940-948.

74. Koncarevic A, et al. A soluble activin receptor type IIb prevents the effects of androgen deprivation on body composition and bone health. Endocrinology. 2010;151(9):4289-4300.

75. Goncalves MD, et al. Akt deficiency attenuates muscle size and function but not the response to ActRIIB inhibition. PLoS One. 2010;5(9):e12707.

76. Camporez JP, et al. Anti-myostatin antibody increases muscle mass and strength and improves insulin sensitivity in old mice. Proc Natl Acad Sci U S A. 2016;113(8):2212-2217.

77. Braga $\mathrm{M}$, et al. Inhibition of in vitro and in vivo brown fat differentiation program by myostatin. Obesity (Silver Spring). 2013;21(6):1180-1188.

78. Fournier B, et al. Blockade of the activin receptor IIb activates functional brown adipogenesis and thermogenesis by inducing mitochondrial oxidative metabolism. Mol Cell Biol. 2012;32(14):2871-2879.

79. Campbell C, et al. Myostatin inhibitor ACE-031 treatment of ambulatory boys with Duchenne muscular dystrophy: results of a randomized, placebo-controlled clinical trial. Muscle Nerve. 2017;55(4):458-464.

80. Tillet E, Bailly S. Emerging roles of BMP9 and BMP10 in hereditary hemorrhagic telangiectasia. Front Genet. 2015;5:456.

81. Wooderchak-Donahue WL, et al. BMP9 mutations cause a vascular-anomaly syndrome with phenotypic overlap with hereditary hemorrhagic telangiectasia. Am JHum Genet. 2013;93(3):530-537.

82. Upton PD, et al. Bone morphogenetic protein (BMP) and activin type II receptors balance BMP9 signals mediated by activin receptor-like kinase-1 in human pulmonary artery endothelial cells. JBiol Chem. 2009;284(23):15794-15804.

83. Nakatani M, et al. Transgenic expression of a myostatin inhibitor derived from follistatin increases skeletal muscle mass and ameliorates dystrophic pathology in $\mathrm{mdx}$ mice. FASEB $J$. 2008;22(2):477-487.

84. Schneyer AL, et al. Differential antagonism of activin, myostatin and growth and differentiation factor 11 by wild-type and mutant follistatin. Endocrinology. 2008;149(9):4589-4595.

85. Bialek P, et al. A myostatin and activin decoy receptor enhances bone formation in mice. Bone. 2014;60:162-171.

86. Chiu CS, et al. Increased muscle force production and bone mineral density in ActRIIB-Fc-treated mature rodents. J Gerontol A Biol Sci Med Sci. 
2013;68(10):1181-1192.

87. Goh BC, et al. Activin receptor type $2 \mathrm{~A}$ (ACVR2A) functions directly in osteoblasts as a negative regulator of bone mass. J Biol Chem. 2017;292(33):13809-13822.

88. Lee SJ, et al. Targeting myostatin/activin A protects against skeletal muscle and bone loss during spaceflight. Proc Natl Acad Sci U S A. 2020;117(38):23942-23951.

89. Roh JD, et al. Activin type II receptor signaling in cardiac aging and heart failure. Sci Transl Med. 2019;11(482):eaau8680.

90. Schang G, et al. Murine FSH production depends on the activin type II receptors ACVR2A and ACVR2B. Endocrinology. 2020;161(7):bqaa056.

91. Garito T, et al. Effects of bimagrumab, an activin receptor type II inhibitor, on pituitary neurohormonal axes. Clin Endocrinol (Oxf). 2018;88(6):908-919.

92. Sun L, et al. FSH directly regulates bone mass. Cell. 2006;125(2):247-260.

93. Liu P, et al. Blocking FSH induces thermogenic adipose tissue and reduces body fat. Nature. 2017;546(7656):107-112.

94. DiGirolamo DJ, et al. Administration of soluble activin receptor $2 \mathrm{~B}$ increases bone and muscle mass in a mouse model of osteogenesis imperfecta. Bone Res. 2015;3:14042.

95. Jeong $\mathrm{Y}$, et al. Skeletal response to soluble activin receptor type IIB in mouse models of osteogenesis imperfecta. J Bone Miner Res. 2018;33(10):1760-1772.

96. Man WD, et al. Quadriceps myostatin expression in COPD. Eur Respir J. 2010;36(3):686-688.

97. Pomiès $\mathrm{P}$, et al. Reduced myotube diameter, atrophic signalling and elevated oxidative stress in cultured satellite cells from COPD patients. J Cell Mol Med. 2015;19(1):175-186.

98. Loumaye A, et al. Role of Activin A and myosta- tin in human cancer cachexia. J Clin Endocrinol Metab. 2015;100(5):2030-2038.

99. Hatakeyama S, et al. ActRII blockade protects mice from cancer cachexia and prolongs survival in the presence of anti-cancer treatments. Skelet Muscle. 2016;6:26.

100.Benny Klimek ME, et al. Acute inhibition of myostatin-family proteins preserves skeletal muscle in mouse models of cancer cachexia. Biochem Biophys Res Commun. 2010;391(3):1548-1554.

101.Zhou X, et al. Reversal of cancer cachexia and muscle wasting by ActRIIB antagonism leads to prolonged survival. Cell. 2010;142(4):531-543.

102. Lang $\mathrm{CH}$, et al. Regulation of myostatin by glucocorticoids after thermal injury. FASEB $J$. 2001;15(10):1807-1809.

103. Ma K, et al. Characterization of 5'-regulatory region of human myostatin gene: regulation by dexamethasone in vitro. Am J Physiol Endocrinol Metab. 2001;281(6):E1128-E1136.

104.Ma K, et al. Glucocorticoid-induced skeletal muscle atrophy is associated with upregulation of myostatin gene expression. Am J Physiol Endocrinol Metab. 2003;285(2):E363-E371.

105. Qin J, et al. Dexamethasone-induced skeletal muscle atrophy was associated with upregulation of myostatin promoter activity. Res Vet Sci. 2013;94(1):84-89.

106. Gilson $\mathrm{H}$, et al. Myostatin gene deletion prevents glucocorticoid-induced muscle atrophy. Endocrinology. 2007;148(1):452-460.

107. Schafer MJ, et al. Quantification of GDF11 and myostatin in human aging and cardiovascular disease. Cell Metab. 2016;23(6):1207-1215.

108. Heineke J, et al. Genetic deletion of myostatin from the heart prevents skeletal muscle atrophy in heart failure. Circulation. 2010;121(3):419-425.

109. Burch PM, et al. Reduced serum myostatin concentrations associated with genetic muscle dis- ease progression. J Neurol. 2017;264(3):541-553.

110. Mariot V, et al. Downregulation of myostatin pathway in neuromuscular diseases may explain challenges of anti-myostatin therapeutic approaches. Nat Commun. 2017;8(1):1859.

111. Cadena SM, et al. Administration of a soluble activin type IIB receptor promotes skeletal muscle growth independent of fiber type. J Appl Physiol (1985). 2010;109(3):635-642.

112. Lee SJ. Regulation of muscle mass by myostatin. Annu Rev Cell Dev Biol. 2004;20:61-86.

113. Lee SJ. Myostatin: regulation, function, and therapeutic applications. In: Hill JA, Olson EN, eds. Muscle: Fundamental Biology and Mechanisms of Disease. Academic Press; 2012:1077-1084.

114. Lee SJ. Extracellular regulation of myostatin: a molecular rheostat for muscle mass. Immunol Endocr Metab Agents Med Chem. 2010;10:183-194.

115. Girgenrath S, et al. Loss of myostatin expression alters fiber-type distribution and expression of myosin heavy chain isoforms in slow- and fast-type skeletal muscle. Muscle Nerve. 2005;31(1):34-40.

116. Zhu Y, et al. LC-MS/MS multiplexed assay for the quantitation of a therapeutic protein BMS986089 and the target protein Myostatin. Bioanalysis. 2016;8(3):193-204.

117. Dagbay KB, et al. Structural basis of specific inhibition of extracellular activation of pro- or latent myostatin by the monoclonal antibody SRK-015. J Biol Chem. 2020;295(16):5404-5418.

118. Morvan F, et al. Blockade of activin type II receptors with a dual anti-ActRIIA/IIB antibody is critical to promote maximal skeletal muscle hypertrophy. Proc Natl Acad Sci U S A. 2017;114(47):12448-12453.

119. Amato AA, et al. Treatment of sporadic inclusion body myositis with bimagrumab. Neurology. 2014;83(24):2239-2246. 